\title{
Sintaxis espacial en el oppidum ibérico. Reflexiones sobre los modelos espaciales y sociales
}

\author{
Space Syntax in the Iberian oppidum. Considerations on space \\ and social models
}

\author{
Ignasi Grau Mira
}

I.U. Investigación en Arqueología y Patrimonio Histórico. Universidad de Alicante e-mail: ignacio.grau@ua.es

\begin{abstract}
RESUMEN
En el presente trabajo realizamos un aproximación mediante el análisis basado en la sintaxis espacial aplicado al espacio doméstico de las sociedades de la edad del Hierro de la franja central de la Iberia Mediterránea, entre los siglos VI y III a. C. En una primera sección se introduce la importancia de la configuración de las casas para comprender la sociedad ibérica. A continuación seleccionamos algunos ejemplos de la mitad meridional del País Valenciano para realizar nuestro análisis. Se identifican de dos modelos espaciales claramente definidos. Por último se relacionan estos esquemas constructivos con los procesos de agregación de unidades familiares para constituir las comunidades ibéricas.
\end{abstract}

Palabras clave: Iberia; Edad del Hierro; Unidades domésticas; agregación social.

\section{ABSTRACT}

In this paper we propose an approach to the analysis based on the space syntax applied to the domestic space of the societies of Iron Age in the central region of Mediterranean Iberia. In the first section, the importance of setting the houses to understand the Iberian society is introduced. Secondly, an analysis of some examples of Mediterranean central area is developed, in which we identify two clearly defined spatial models. Finally these models are related to the aggregation of households to make up the Iberian communities.

Keywords: Iberia; Iron Age; households; social aggregation.

Recibido: 19-05-2015. Aceptado: 02-07-2015.

Cómo citar este artículo / Citation

Grau Mira, I. 2015: "Sintaxis espacial en el oppidum ibérico. Reflexiones sobre los modelos espaciales y sociales ", Arqueología de la Arquitectura, 12: e032. doi: http://dx.doi.org/10.3989/arq.arqt.2015.123

\section{Copyright}

(c) 2015 CSIC. Este es un artículo de acceso abierto distribuido bajo los términos de la licencia Creative Commons Attribution-Non Commercial (by-nc) Spain 3.0. 


\section{INTRODUCCIÓN: LA CASA EN EL OPPIDUM IBÉRICO}

La inclusión de un estudio referido al mundo ibérico en este volumen dedicado al análisis espacial nos permite reflexionar sobre algunas de las propuestas de organización social que encuentran en los modos de articulación espacial no solo un reflejo, sino también el medio de la acción humana. Es decir, tomamos la perspectiva de entender el diseño arquitectónico desde una perspectiva sociológica que atienda a los comportamientos y usos sociales del espacio. Así, el espacio construido transmite normas, mediante mensajes de índole no verbal, que van indisolublemente asociadas al diseño formal de las casas y especialmente la segmentación y las relaciones de los distintos espacios (Rapoport 1978; Kent 1990; Allison 1998).

Nuestro propósito no es hacer un riguroso estudio, basado en un exhaustivo corpus de unidades domésticas, que excedería las posibilidades de un artículo. Más bien queremos mostrar las capacidades heurísticas del análisis basado en la sintaxis espacial y reflexionar sobre los modelos sociales que pueden interpretarse a partir de los esquemas espaciales presentes entre los grupos ibéricos de la franja central mediterránea.

Nuestras primeras líneas, y antes de entrar en las particularidades de nuestro análisis, van a ir dirigidas a señalar el alcance y condicionantes de la aproximación que desarrollaremos en este trabajo. La primera consideración es la referida a la propia área ibérica en la que se centra este estudio. Iberia no fue una unidad política hasta que la llegada de Roma unificó los territorios bajo su dominio. Más bien nos referimos a un conjunto cultural formado por grupos relativamente autónomos que hacia la época plena, principalmente en el s. III a. C., se organizaron en entidades territoriales presididas por asentamientos urbanos (Ruiz 2007) que pudieron reunirse temporalmente bajo la forma de federaciones regionales.

Dentro de este amplio mosaico político, en este trabajo vamos a centrarnos en el área central de la fachada mediterránea ibérica (fig. 1), y que en la actualidad conforman las tierras del centro y el sur del País Valenciano. Esta región cuenta con rasgos que le confieren uniformidad cultural y de organización social, aunque acogería distintas unidades políticas en su seno (Bonet, Grau y Vives-Ferrándiz 2015). Esta zona se distinguiría de otras áreas al sur y al norte de Iberia, donde los modelos sociales presentan sus propias particularidades (Ruiz 2007; Sanmartí 2004; Sanmartí y Belarte 2001).

Debemos señalar las limitaciones del registro arqueológico referido a los espacios de hábitat ibéricos, donde en pocas ocasiones contamos con amplias áreas excavadas que permitan el análisis de plantas completas. Escogemos para nuestro estudio grandes asentamientos fortificados que podemos caracterizar como oppida o centros de poder locales, donde su amplia extensión y la variabilidad de la población nos permiten encontrar grupos sociales diversos y su expresión en los ámbitos domésticos.

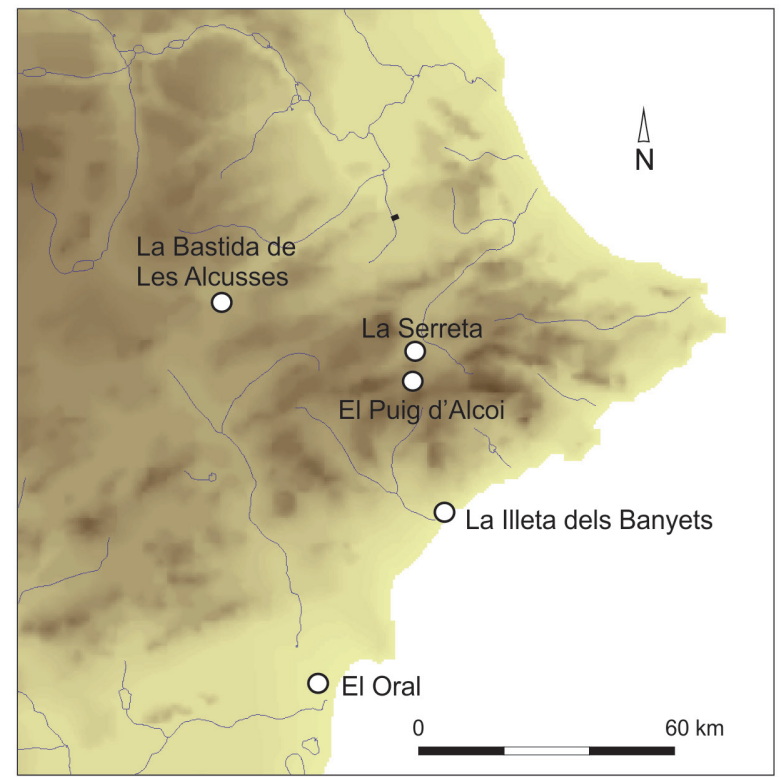

Figura 1.- Localización del área de estudio con los sitios analizados: 1: La Bastida de les Alcusses, 2: La Serreta, 3: El Puig d’Alcoi, 4: La Illeta del Campello, 5: El Oral. 
Durante el primer milenio a. C. en Iberia acaecen procesos de urbanización y centralización de la población en oppida. Para la mayor parte de la población dedicada a la agricultura y el pastoreo, la vida urbana y concentrada supone una desventaja, especialmente por la necesidad de desplazarse desde el lugar de residencia a los campos y pasturas. Este inconveniente se acentúa cuando el emplazamiento de los oppida busca las cimas de los cerros, en ocasiones con largos y penosos desplazamientos hasta las tierras de labor. En ese sentido, la existencia de asentamientos dispersos próximos a las áreas de cultivo y espacios de trabajo configuraría un patrón espacial mucho más eficiente desde el punto de vista productivo. De esta evidencia se deriva que el proceso de formación del modelo ibérico del oppidum está relacionado con procesos sociales, económicos y políticos de hondo calado. De ese modo, este tipo de asentamientos se convierte en el escenario principal de los juegos de poder y los procesos de configuración de la sociedad ibérica y por ello nuestro análisis se centrará en las casas de estos centros principales.

En estos oppida, pese a reconocerse viviendas de elites y grupos subordinados (Belarte, Bonet y Sala 2009), no existe una gran complejidad en la configuración de las unidades domésticas, antes bien son viviendas muy sencillas, como a continuación veremos. Esas características en principio abogarían en contra del uso de análisis sintáctica que encuentra todo su potencial en estructuras domésticas complejas del mediterráneo antiguo, como la casa romana (Bermejo 2013). Sin embargo, ofrece interesantes posibilidades que vamos a tratar de explorar.

\section{LA IMPORTANCIA DE LA CASA EN LA SOCIEDAD IBÉRICA}

El estudio de las estructuras sociales de época ibérica puede abordarse desde planteamientos y fundamentos variados, orientados por posiciones teóricas diferentes, que enfatizan distintos aspectos de la organización social. Por lo general, los estudios referidos a los iberos coinciden en señalar que habían alcanzado formas de organización social definidas como jefaturas complejas (Aranegui 2012) alcanzando formas estatales (Ruiz 2007; Grau 2007). Aunque estos estados ibéricos no habrían desarrollado algunos de los rasgos de sociedades estatales clásicas, lo que los aproximaría a las estructuras definidas como estados arcaicos, con escaso desarrollo institucional (Sanmartí 2004).

La documentación disponible expresa con cierta claridad este limitado desarrollo de algunas estructuras estatales clásicas. En los escasos textos grecolatinos no existe una mención explícita de estado al referirse a los iberos. Tampoco los rasgos arqueológicos mostrarían claramente las instituciones propiamente del estado, como son el palacio y el templo. Aunque pueden reconocerse algunas residencias destacadas y lugares de culto, no tienen la consideración de centros articuladores de la administración y la actividad económica, como sucede en otras culturas. Tampoco existen otras instituciones como burocracia y ejércitos permanentes. De ello se deriva que nos encontramos con formas poco formalistas de estado clásico, que suelen definirse como estados arcaicos, estructuras en las que las formas sociales pre-estatales tendrían mucho peso: el lenguaje del linaje, las formas personales de asunción del poder, los matrimonios, las relaciones de parentesco de algunos linajes, etc.

En segundo lugar, en el ámbito ibérico la mayor parte de las actividades económicas de especialización quedan circunscritas al ámbito doméstico. Buena parte de los talleres e infraestructuras artesanales, los equipamientos de transformación agraria, como lagares y almazaras, los almacenes... se vinculan a las casas y espacios domésticos. Algunos ejemplos destacados como La Bastida de les Alcusses ofrecen una expresión clara de este esquema organizativo (Vives-Ferrándiz 2013).

Por todo ello, adquiere un especial interés explorar las bases de la organización económica y social en el seno de los grupos domésticos ibéricos. Recientes trabajos han fijado su atención en como la casa, como estructura física, y la Casa, como unidad social según el concepto de Lévi-Strauss, ofrecen una aproximación de gran valor para entender la sociedad ibérica (VivesFerrándiz 2013). De ese modo la agencia, o capacidad transformadora y articuladora de las formas sociales, adquiere relevancia en relación al nivel del grupo doméstico (el household en términos anglosajones) y también las asociaciones de los grupos corporativos, para ir estructurando posteriormente los niveles de instituciones superiores.

De acuerdo a estos planteamientos, nos centramos en este trabajo en el análisis de la estructura de estas unidades domésticas, su relación entre ellas y con los procesos de transformación como evidencia de las dinámicas sociales de los grupos ibéricos. 


\section{PROPUESTA TEÓRICA: PRÁCTICA, SINTAXIS ESPACIAL, INTERACCIÓN SOCIAL}

Las propuestas teóricas derivadas de la agencia se basan en la insatisfacción producida por las aproximaciones sistémicas que al centrarse en la estructura de la sociedad han obviado el comportamiento de la gente. El postulado básico es que las personas no son autómatas sin conciencia que reaccionan a los estímulos externos, sino al contrario intervienen plenamente en la reproducción de las estructuras sociales. Las personas son conscientes de las normas sociales y pueden actuar creativamente en el seno de estas estructuras, aunque están constreñidos por estas reglas y sólo excepcionalmente pueden subvertir el orden social establecido (Dobres y Robb 2000, 2005; Dornan 2002).

Esta aproximación es especialmente pertinente para orientar la investigación de los espacios domésticos y las prácticas cotidianas. La configuración de las casas es un ámbito de estudio que permite referirnos a la existencia de normas y reglas, pero también las posibilidades de modificación. Así mismo, en este ámbito se expresan los modos de interacción social en el contexto de lo cotidiano.

Desde el punto de vista metodológico nos proponemos desarrollar el estudio combinado de las funciones de los espacios domésticos que se deduce por los equipamientos y ajuares recuperados, teniendo en cuenta los condicionantes propios de la formación del registro, y la estructura espacial de la casa. Vamos a analizar la circulación en las estructuras construidas mediante el denominado justified gamma map, mapa gamma justificado (Hillier y Hanson 1984: 149), también denominado 'mapa jerárquico' según la terminología de Blanton (1994: 37). Se trata de una representación gráfica que muestra por medio de líneas las diferentes conexiones entre unidades espaciales, el conjunto de la edificación se pone en relación al espacio exterior, que se representa como un círculo con un aspa, que se sitúa en la base del gráfico (Bermejo 2009: 53). Con ello se permite reconocer los modos de conexión topológica, o relación entre espacios, como el gradiente de penetración (Rapoport 1978: 289-298) o de profundidad estructural (Blanton 1994: 37), es decir, las posibilidades de controlar la circulación entre el exterior y el interior de las viviendas.

La principal aportación de una aproximación de este tipo es la descripción de genotipos arquitectónicos reconocibles en las viviendas ibéricas. Si bien la principal característica de la arquitectura doméstica es su gran heterogeneidad, lo que dificulta la observación de modelos normativos (Belarte 2013; Belarte, Bonet y Sala 2009; Grau 2013; Sala 2005), el empleo de estas herramientas analíticas de la sintaxis espacial permite detectar la existencia de recurrencias topológicas. Esto significa que dentro de la variedad de plantas y organización de las estancias, se pueden detectar las formas de organizar las relaciones espaciales que subyacen al diseño de las construcciones. Nos interesa especialmente identificar las formas potenciales de regular la circulación a través de espacios transicionales y, a partir de ellas, explorar las normas y preceptos sociales que subyacen a la producción y uso del espacio.

Para ilustrar nuestro procedimiento acudimos a un ejemplo que expresa claramente como viviendas muy semejantes reflejan esquemas espaciales claramente distintos. Se trata de las casas del sector III del asentamiento ibérico antiguo, fines del s. VI y s. V a. C., de El Oral (San Fulgencio, Alicante) (fig. 2), descritas y analizadas en trabajos de detalle que aportan la información necesaria para el análisis (Abad y Sala 1993; Abad, Sala, Grau, Moratalla, Pastor y Tendero 2001).

Nos interesan las casas IIIC, IIID y IIIG, cuyas estructuras a simple vista reproducirían un esquema similar. Las dos primeras son viviendas prácticamente idénticas formadas por una estructura rectangular que se divide en dos departamentos: uno principal que dispone de hogar y que sería el área de vivienda, y un segundo espacio ligeramente menor y situado en la parte posterior que posiblemente es un área de almacenaje (Abad y Sala 1993: 60). Ambas tienen una superficie muy semejante, IIIC se extiende por $17,7 \mathrm{~m}^{2}$ y IIID por 17,3 $\mathrm{m}^{2}$ (Abad y Sala 1993: 165). Por su parte, la casa IIIG en su fase inicial, que ahora nos interesa, configura una vivienda inscrita en un rectángulo que repite el esquema anteriormente descrito de casas simples de forma simétrica, pero con el añadido de un vestíbulo en forma de $\mathrm{T}$, el espacio IIIG1, que separa el exterior del interior (Abad, Sala, Grau, Moratalla, Pastor y Tendero 2001: 159). Posteriormente se añade a la vivienda inicial un nuevo cuerpo formado por dos departamentos que se añaden a la zona postrera de la vivienda. El conjunto se interpreta como una casa que acoge a dos familias nucleares que incorpora una tercera por el crecimiento del grupo familiar (Abad y Sala 1993: 60).

De ese modo, tendríamos en origen dos conjuntos: uno formado por la unión de IIIC-IIID, y el de la casa IIIG. Cada uno de ellos acoge en la primera fase dos 
unidades domésticas muy semejantes por la replicación de la estructura de casa básica y con espacio de vivienda, caracterizado por la presencia del hogar, cuyas superficies son prácticamente idénticas: IIIC1 con $10,85 \mathrm{~m}^{2}$, IIID1 con $10,40 \mathrm{~m}^{2}$, IIIG2 con $10,75 \mathrm{~m}^{2}$ y IIIG3 con $13,55 \mathrm{~m}^{2}$.

Ahora bien, el análisis de la sintaxis espacial (fig. 2) nos permite observar que la identidad de las unidades domesticas básicas desparece en la forma de agregación de estos módulos. La casa IIIG muestra un esquema agregado y replegado en sí mismo, con el núcleo del hogar en las partes profundas de la casa y cuyo acceso se mediatiza con espacios transicionales, en busca de una separación del exterior. Mientras que las otras casas se encuentran disociadas y abiertas al exterior, sin espacios intermedios entre la calle y el núcleo de la vivienda con el espacio del hogar.

\section{LAS CASAS BAJO EL ANÁLISIS ESPACIAL}

Vamos a extender el análisis que nos ha servido de ejemplo a otras unidades domésticas del área de estudio y del mismo ámbito sociocultural.

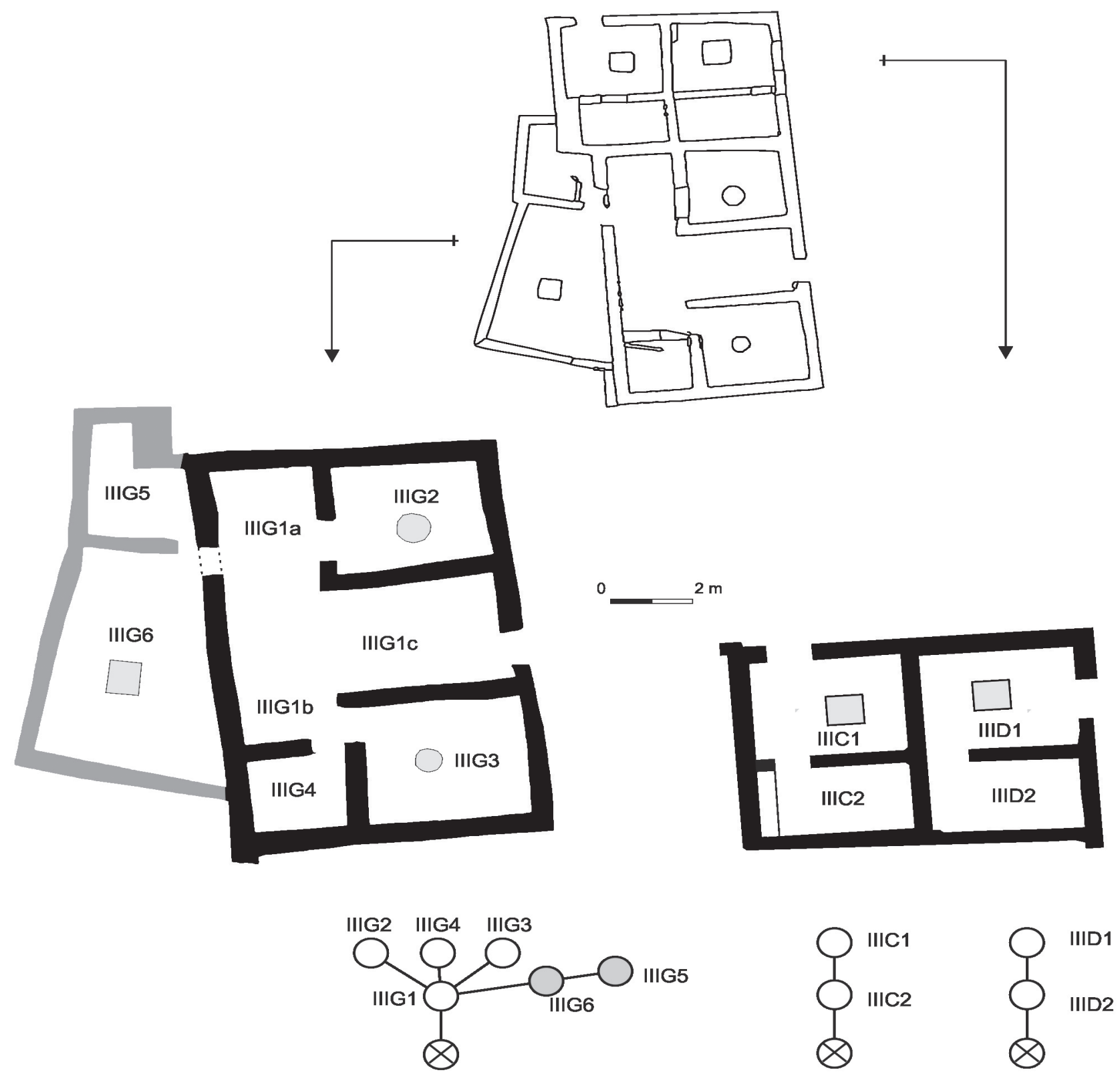

Figura 2.- Planta y mapa gamma justificado de las casas IIIC, IIID y IIIG de El Oral (Elaboración propia a partir de Abad, Sala, Grau, Moratalla, Pastor y Tendero 2001). 


\subsection{Las casas con espacio de transición: el control de los contactos}

Empezando por el mismo poblado de El Oral, nos encontramos con otros ejemplos que seguirían el mismo esquema topológico caracterizado por el esquema arboriforme con un 'espacio de transición externo', es decir, un espacio transicional que puede ser un vestíbulo, un corredor o un patio.

Una de estas casas es la denominada IVH (Sala y Abad 2006: 30-31) (fig. 3). Se trata de una gran vivienda de $209 \mathrm{~m}^{2}$ que se inscribe en un trapecio adosado a la muralla de cierre del poblado. Tiene una entrada en codo por el extremo sudoeste que da acceso a un gran patio IVH6 que distribuye la circulación a varias zonas. Al norte del patio se encuentra un espacio IVH7 con un gran hogar central que evidencia un ámbito con actividad de transformación. Adjunto a este espacio se localiza otro hogar en un espacio sin individualizar del patio. La habitación principal de la casa se encuentra al oeste del patio en una posición central, es el espacio IVH8, la sala noble de la casa (Sala y Abad 2006: 31), que se opone a una batería de estancias que forman el ala oriental. Son las estancias IVH5, IVH4, IVH3 y IVH2, ésta última con un vestíbulo que la antecede, IVH1. Estos departamentos se interpretan como salas de reposo, o incluso con funciones de almacén (Sala y Abad 2006: 31).

Otra de las casas que responden al esquema descrito es la denominada IVA (Sala y Abad 2006: 31-32) (fig. 4). De nuevo es una casa cuya amplia entrada da lugar a un gran patio, IVA1, que es el foco de actividad principal de la casa y el espacio distribuidor de la circulación. Desde este patio se accede a tres estancias muy semejantes, caracterizadas por testimonios limitados de actividad, la existencia en dos de ellas de hogares, las IVA2 y IVA3, y con equipamientos y acabados domésticos cuidados en las tres. Se interpretan como espacios de reposo o reunión, y posible función mixta de despensa (Sala y Abad 2006: 32).
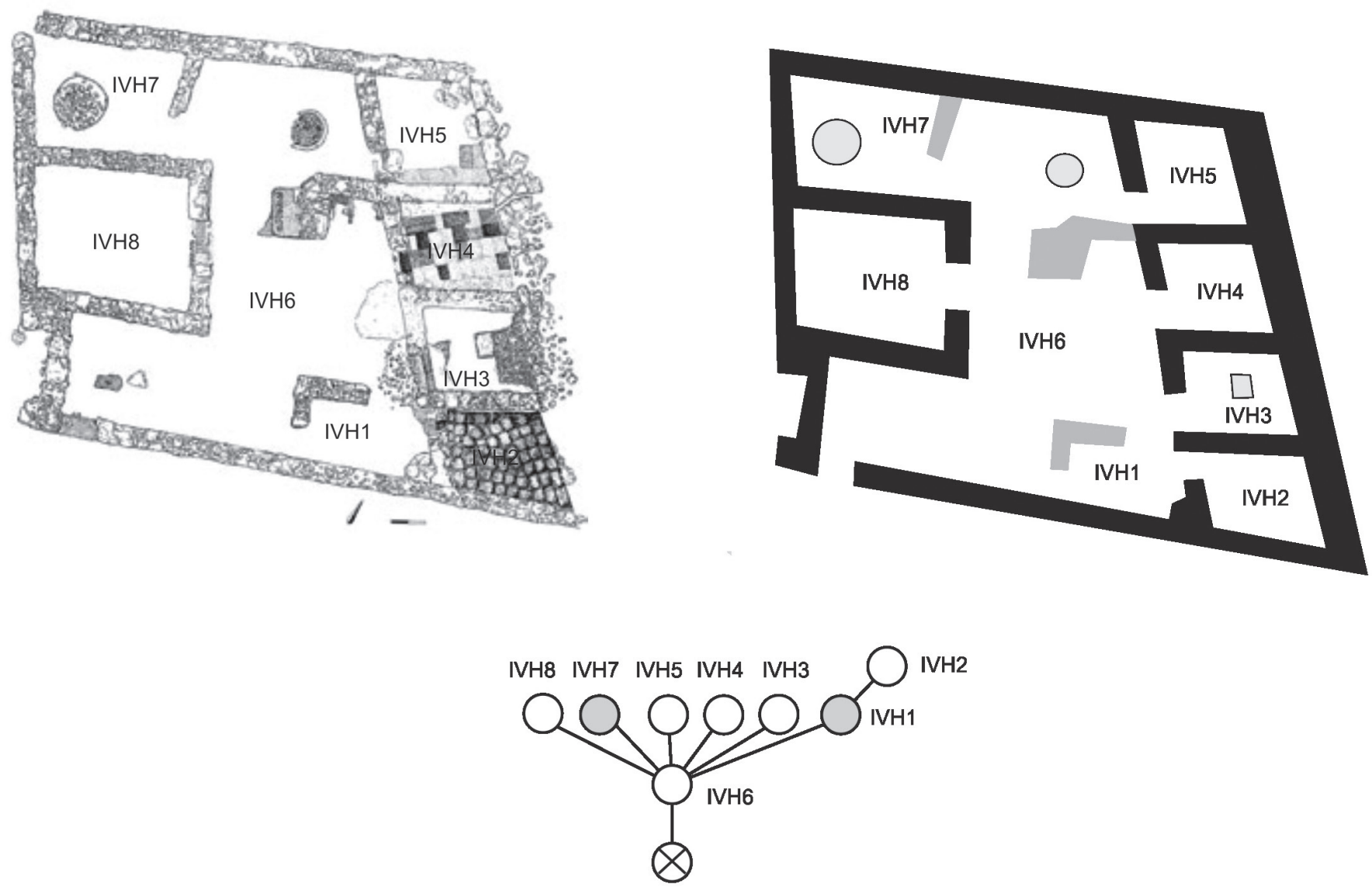

Figura 3.- Planta y mapa gamma justificado de la casa IVH de El Oral (Elaboración propia a partir de Sala y Abad, 2006). 
Como vemos, estas viviendas se dotaron de estos dispositivos liminales o espacios de transición que tienen una naturaleza dual, pues pueden generar un lugar de encuentro entre el exterior y el interior de la casa, pero por otra parte tienen el potencial de dividir el espacio, las actividades y la gente (Hanson 1998: 285). El uso de este ambiente liminal tiene la función de evitar intrusiones no deseadas en el foco de vivienda, mediante la reducción de la permeabilidad al foco del hogar, y también actúa como una señal eficiente que emite el mensaje de la división de la actividad exterior y el dominio interior de la casa.

La existencia de estos espacios de transición evidencia dos hechos que debieron estar interconectados. El primero es que las relaciones entre los miembros del interior y del exterior de la unidad doméstica debieron ser lo suficientemente frecuentes como para crear la necesidad de regularlos mediante dispositivos arquitectónicos. Y el segundo aspecto, conectado con el primero, es que las relaciones necesariamente debían estar sometidas a reglas específicas, cuyas propiedades sociales se canalizaron a través del espacio de transición. Se produjo, de ese modo, una configuración espacial en forma de árbol que enmarca, distingue y articula circulaciones y categorías de gente y actividades en un patrón de encuentros controlados (Hillier y Hanson 1984: 166-167).

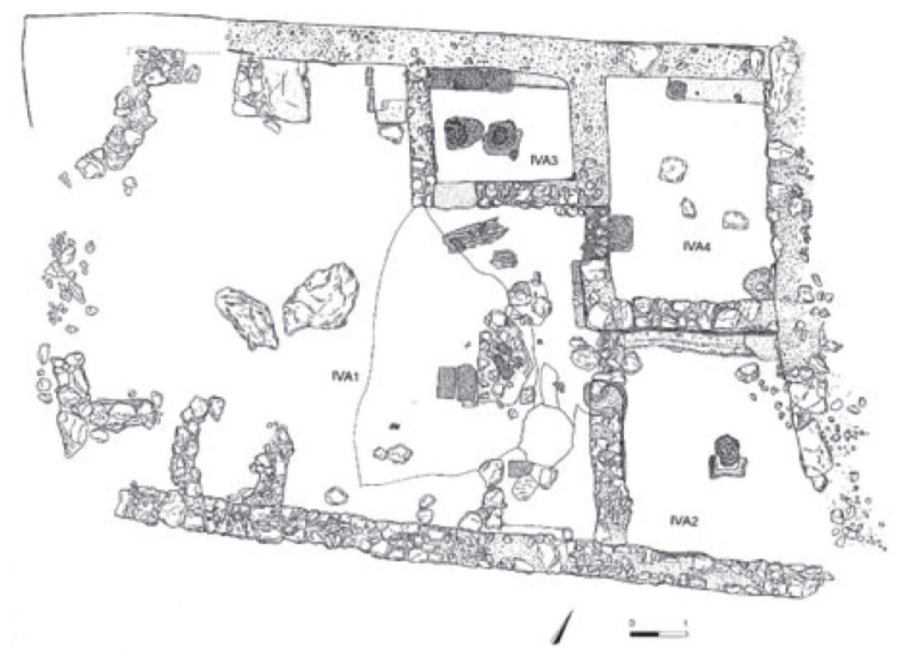

\subsection{Las casas sin espacio de transición: la permeabilidad del contacto exterior}

En el mismo poblado del Oral se reproduce el genotipo de casa sin espacios transicionales que hemos descrito al referimos a IIIC y IIID, cuyo esquema extremadamente simple no permite ningún tipo de articulación de la circulación. Sin embargo, otras casas más complejas muestran un esquema homologable y que se caracterizaría por carecer de control sobre los encuentros entre los habitantes de la casa y las personas ajenas. Tal es así, que al espacio del hogar, núcleo de la vivienda, se accede directamente desde el exterior. Ese es el caso de la vivienda IIIK (fig. 5) una de las más grandes y complejas del poblado, especialmente debido a las ampliaciones que se sucedieron en la casa (Abad, Sala, Grau, Moratalla, Pastor y Tendero 2001: 103; Sala y Abad 2006: 34).

En su estructura original, desde el exterior se accede a la habitación IIIK9, cuyos equipamientos y ajuares, con el hogar central, la convierten en el núcleo de actividad doméstica de la casa (Sala y Abad 2006: 34-35). También desde el exterior se accede a IIIK5, que es una sala que ejerce de distribuidor hacia las habitaciones al este y hacia IIIK6, una habitación con acabados cuidados y un banco corrido. El ala oriental se interpreta como la zona de trabajo y almacén de la vivienda (Abad,
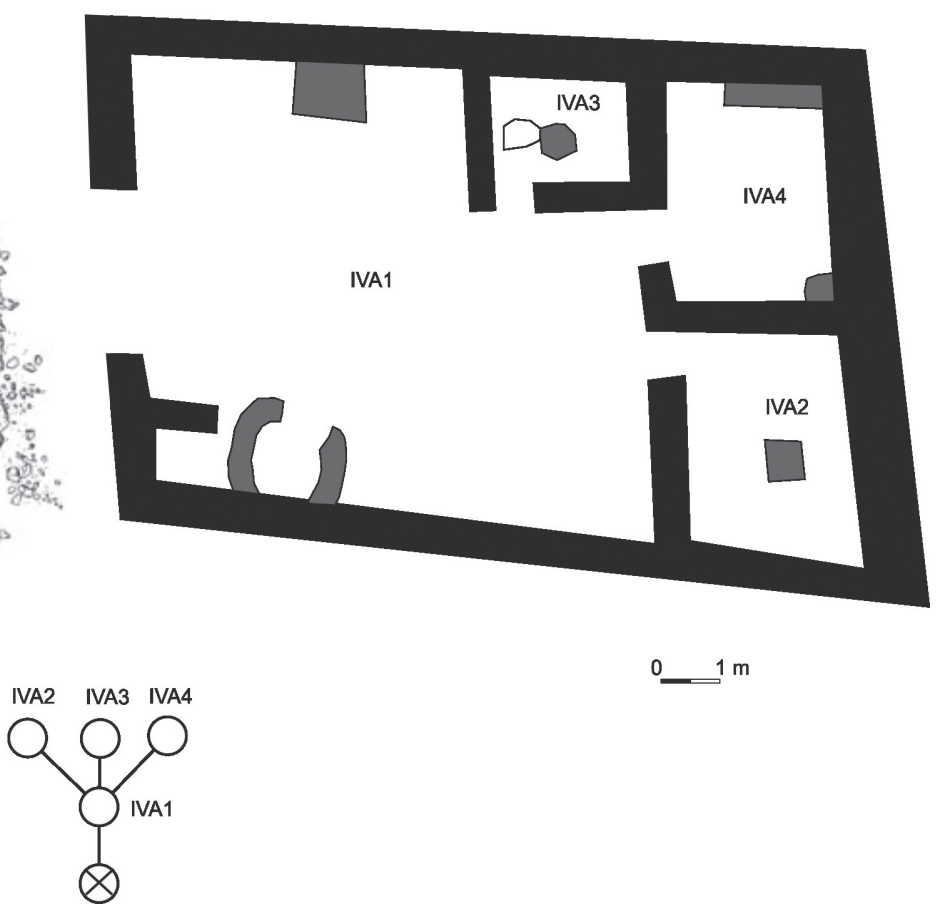

Figura 4.- Planta y mapa gamma justificado de la casa IVA de El Oral (Elaboración propia a partir de Sala y Abad, 2006). 
Sala, Grau, Moratalla, Pastor y Tendero 2001: 163). Está formada por una habitación principal IIIK1 que da paso a IIIK2 y desde ésta a dos habitáculos profundos de menores dimensiones IIIK3 y IIIK4.

Esta vivienda, a pesar de sus grandes dimensiones, su compleja estructura y sus sucesivas ampliaciones, no transformó el esquema básico de ofrecer una gran permeabilidad al núcleo de actividad de la vivienda, con el espacio del hogar accesible directamente desde la calle, pauta que se repite con la ampliación de un nuevo espacio de hogar con IIIK8.

De ese modo, nos encontramos el mismo genotipo en esta casa compleja y en las casas de esquema simple como son las ya descritas IIIC y IIID, y también en otras del mismo poblado como las IIB, IVE y IVF (Abad, Sala, Grau, Moratalla, Pastor y Tendero 2001). Podemos deducir que más allá de la disponibilidad de espacio, complejidad de las casas y su posible asociación a familias con estatus elevados, como podría ser el caso de IIIK, encontramos que en el diseño escogido en ningún momento priorizó el control del acceso al núcleo de la vivienda y obvió la creación de un esquema arbóreo con un espacio de transición frontal.

Este mismo esquema es el que más frecuentemente encontramos en otros casos de viviendas complejas en el área de estudio. Para ilustrar este aserto acudimos a ejemplos de la zona que corresponden a momentos posteriores, centrados en el s. IV a. C., por lo que se podría aludir que corresponden a otro periodo ibérico. Sin embargo, en términos sociales son apenas dos, quizá tres generaciones posteriores a las casas del Oral, por lo que la proximidad temporal sostiene la comparación.
Una casa cuya comparación con las de El Oral es especialmente interesante es la casa ibérica de la manzana 3 de La Illeta dels Banyets de Campello, datada en el s. IV a. C. Ambos asentamientos son enclaves litorales con una base económica claramente orientada hacia los intercambios, según se deduce de las particularidades de sus emplazamientos y los registros materiales que han proporcionado (Abad, Sala, Grau, Moratalla, Pastor y Tendero 2001; Olcina 2005).

La vivienda del Campello forma parte de un complejo edificado formado por dos unidades constructivas. En el sector sureste se dispone un bloque interpretado como área de trabajo y transformación agrícola, en concreto un lagar y otras instalaciones (Olcina 2005: 154155) a las que no aludiremos en este momento. Adosada a esta unidad del sureste se construye un bloque que se interpreta como una vivienda inscrita en un rectángulo con una superficie de $78 \mathrm{~m}^{2}$ (fig. 6). Al sur se dispone un patio semi-cubierto de $34 \mathrm{~m}^{2}$ al que se accede desde la calle y también desde el núcleo de la vivienda formado por los departamentos 27, 28, 30 y 31 . El foco de la casa, señalado por la presencia del hogar se localiza en el dep. 30 (Olcina 2005: 156; Olcina, Martínez y Sala 2009).

Interesa señalar que el esquema topológico de la vivienda muestra un contacto con el exterior sin mediatizar por un espacio conector de forma arbórea, como en las casas de patio de El Oral. Aunque la estructura de la casa hubiese permitido anteponer este espacio de transición, se prefirió abrir el núcleo de la vivienda directamente a la calle, aunque el espacio del hogar aparece mediatizado por un departamento intermedio.
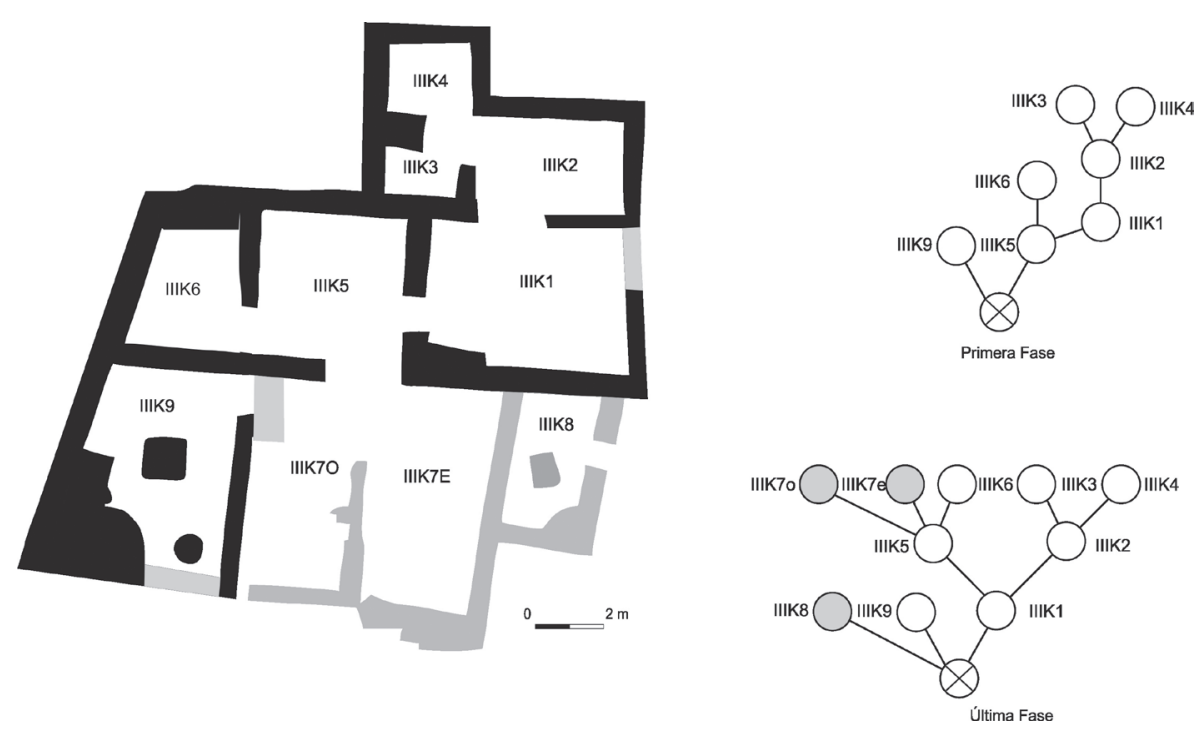

Figura 5.- Planta y mapa gamma justificado de la casa IIIK de El Oral (Elaboración propia a partir de Sala y Abad, 2006). 


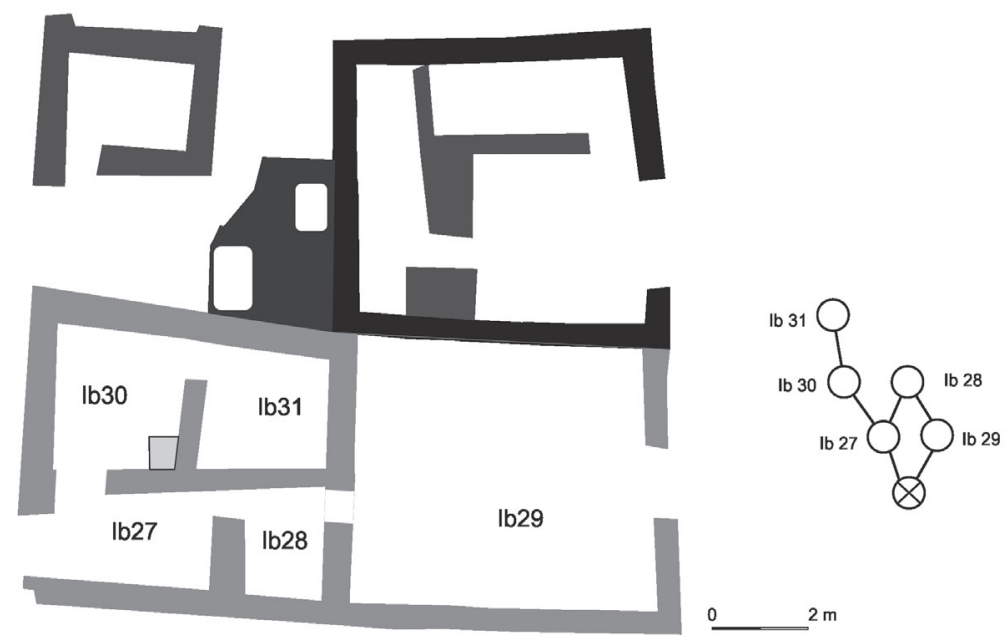

Figura 6.- Planta y mapa gamma justificado de la casa de La Illeta dels Banyets (Elaboración propia a partir de Olcina, Martínez y Sala 2009).

Traemos a nuestra discusión la casa 10 del conjunto 4 del asentamiento de La Bastida de les Alcusses (Moixent, Valencia) (fig. 7). Esta vivienda compleja del s. IV a. C. en un primer estudio se interpretó como un posible conjunto palacial con las particularidades propias del mundo ibérico, es decir, sin una arquitectura especialmente destacada. Los argumentos que hacían destacar esta vivienda de las restantes del poblado eran su tamaño excepcional, la calidad de sus ajuares y la calidad de sus acabados domésticos, con el uso de la cal y decoraciones interiores (Díes y Álvarez 1998: 337).

Una reciente revisión de este conjunto propone que en realidad se trata de dos bloques independientes separados por un espacio abierto (Depto. 211) (Belarte,
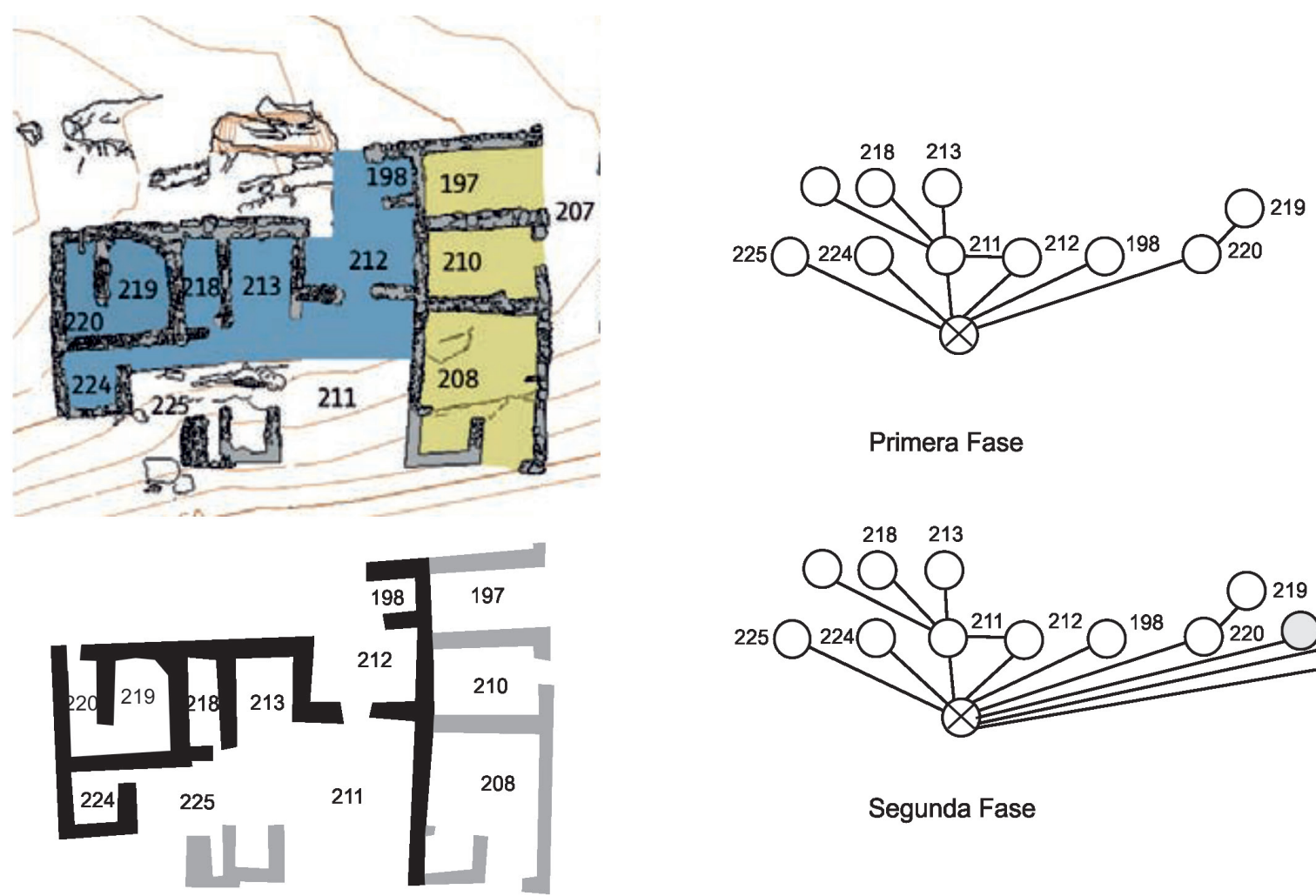

Primera Fase

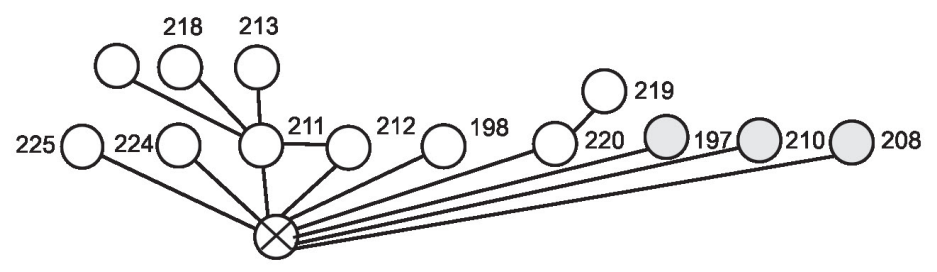

Segunda Fase

Figura 7.- Planta y mapa gamma justificado de la casa de La Bastida de les Alcusses (Elaboración propia a partir de Bonet, Soria y Vives-Ferrándiz 2011). 
Bonet y Sala 2009: 115; Bonet, Soria y Vives-Ferrándiz 2011: 142-143). La parte oriental de la construcción está formada por tres departamentos $(197,208$ y 210) con accesos independientes desde la calle (Bonet, Soria y Vives-Ferrándiz 2011: 143). La parte occidental se conforma por dos partes independientes: un sector con acceso directo desde la calle formado por los deptos. 219 y 220; y otro cuerpo formado por los deptos. 213, 218, 224 y 225 , a los que se accede desde la calle a través del área abierta 212. En esta parte oriental carecemos de información respecto a la ubicación de los hogares, lo que limita el alcance de las interpretaciones, pero destacan los ajuares localizados en los departamentos 218 y 219, que podrían corresponder con sendos espacios de vivienda. En el 218 se halló la célebre figura de bronce que representa un guerrero a caballo.

Así las cosas, este conjunto pudo estar compuesto por un agregado de varias unidades domésticas, al menos una formada por los departamentos 219 y 220 , y otra formada por los deptos. 212, 213, 218, 224 y 225. En ellos se encontraría un departamento más profundo, quizá el núcleo de la vivienda, al que se accede por un espacio interpuesto. A ellos habría que sumar los espacios anexos de la parte oriental.

La casa-agregado B de El Puig d'Alcoi (Grau y Segura 2013: 177-190) ofrece un esquema semejante (fig. 8). Esta vivienda tiene su origen a fines del s. V a. C., cuando se construye una casa inscrita en un rectángulo y que cuenta con sendas habitaciones con dimensiones entre $20-25 \mathrm{~m}^{2}$, cada una con acceso independiente y con un hogar en su parte central, lo que sugiere que se trata de dos viviendas habitadas por sendas familias íntimamente relacionadas, pues cooperaron en la construcción unitaria de la casa. La simplicidad de la estructura doméstica en esta primera fase imposibilita la segregación de espacios y encontramos una conexión directa entre el espacio de la calle y la habitación del hogar, auténtico foco de la vivienda.

Una generación después, a inicios del s. IV, se amplía la vivienda con la construcción de las cuatro estancias hasta configurar un agregado doméstico de aproximadamente $100 \mathrm{~m}^{2}$, unas considerables dimensiones para la norma del poblado. Se construyen dos estancias, la 5000 y la 11000 , de dimensiones en torno a $20 \mathrm{~m}^{2}$ con hogares centrales y repertorios cerámicos variados que sugieren que nos encontramos con estancias plurifuncionales que pudieron operar como viviendas de familias nucleares. A ellos se suman un espacio de trabajo, dep. 7000, y un almacén, 2000, que debieron ser comunes.

El gráfico de accesibilidad de las viviendas de la nueva fase sigue mostrando un esquema disgregado, aunque se puede reconocer que las estancias del hogar, foco de la vida social de las familias se reservan un mínimo de privacidad. Para ello se interponen espacios domésticos entre la calle y el departamento del hogar, con lo que el grado de permeabilidad se reduce. La limitación de las relaciones físicas entre los hogares y la calle se establece en el ámbito 5000 con la existencia entre medio del ámbito 7000. Mucho más evidente es el caso de la estancia 6000 en que se construye un tabique que separa un corredor, 11000, a modo de vestíbulo que se interpone entre la calle y el hogar y ejerce de pantalla visual.

\section{B

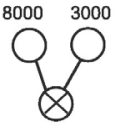

Primera Fase

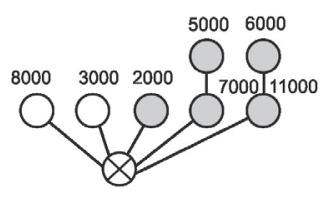

Segunda Fase
10020
5000 3000

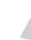

7000

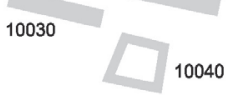

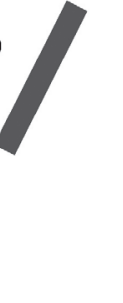

$\underline{0}$
Figura 8.- Planta y mapa gamma justificado de la casa de El Puig d'Alcoi. 


\section{INTERPRETACIONES TENTATIVAS Y REFLEXIONES SOBRE EL ESQUEMA ESPACIAL}

La identificación de esta diferente relación topológica en las formas de agregación de unidades familiares básicas abre las posibilidades de proceder al análisis comparativo, y extender el número de ejemplos y casas en busca de recurrencias estructurales que podamos asociar a ambos genotipos. Y lo que es más importante, proponer interpretaciones en términos sociales del porqué de ambas estructuras.

Conviene empezar sintetizando de forma sumaria los rasgos de los dos esquemas espaciales claramente diferenciados.

a) Un modelo en que se identifica un espacio de transición entre el exterior y el interior. Este ámbito permite dividir y también integrar a los distintos miembros de la unidad doméstica, y a estos con los visitantes ajenos. Sugiere la necesidad y voluntad del control de los encuentros y las dinámicas sociales que requieren situaciones de co-presencia. Al construir las casas con estos espacios liminales, se generó un patrón de movimiento y ocupación que claramente condicionó los encuentros entre los miembros de la casa y los externos: favoreciéndolos, controlándolos o evitándolos.

En un trabajo anterior (Grau 2013) hacíamos alusión a que este esquema arbóreo, con el espacio de transición, correspondía a viviendas multifocales. Se caracterizan por la replicación coherente de habitaciones idénticas, muchas de ellas dotadas de hogares, en una unidad arquitectónica más amplia, lo que se asociaría a residencias multifamiliares, posiblemente formadas por familias extensas.

Estas familias escogen el modelo replegado en el que la experimentación del espacio favorece el desarrollo de distancias sociales entre los residentes y el mundo exterior. Es decir, desde los momentos de socialización en la infancia se está inculcando que existe una clara diferencia entre el espacio de la familia y el de los individuos ajenos a ella. Tales normas de conducta se adquieren mediante una experiencia física del espacio (Bourdieu 1972). En definitiva, la segmentación de espacios facilitaría la codificación de pautas de comportamiento y un claro sentido de las esferas públicas-expuestas y privadasencubiertas.

b) Otro modelo de casas se define porque en ningún momento sus espacios se integraron a partir de un espacio de transición interior, a pesar de su complejidad estructural y de disponer de varios departamentos adosados. El movimiento es exclusivamente exterior. Las viviendas disponen de varias entradas a espacios segregados. En ocasiones se interpone un habitáculo entre la calle y el espacio del hogar, pero en otras ocasiones se accede directamente a este foco principal de la vivienda. De ello se deduce que no hay una decidida búsqueda de separar el grupo residente del ajeno, fomentando la distancia social, como sí ocurría en el caso anterior.

La pauta recurrente de ordenamiento del espacio, con escasas posibilidades de segregación del hogar y el predominio de la vida expuesta, sugiere un intenso contacto entre los grupos familiares. El favorecimiento de esta interrelación debió potenciar las posibilidades de formación de los grupos corporativos ibéricos en el nivel superior a la propia familia nuclear. A nuestro parecer, esa capacidad de trascender el grupo consanguíneo para conformar nuevos grupos de carácter suprafamiliar debió ser un elemento clave en la formación de la sociedad ibérica y por ello se dio la preferencia de unidades fácilmente asociables.

Se podría argüir que en estas casas agregadas pero con circulación exterior nos encontramos simplemente con un conjunto de viviendas independientes, lo que justificaría la falta de conexiones interiores. Sin embargo, hay dos rasgos que definen la configuración del espacio y abogan por su consideración como casas interrelacionadas. El primero es que la construcción de habitaciones o unidades es dependiente de la existencia de una arquitectura preexistente, sobre cuyos muros descansa la ampliación. La segunda es la preferencia que muestran en la ampliación de una estructura preexistente en vez de crear una unidad separada, independiente, en una zona del solar del poblado a pesar de que hubiese espacio para ello.

Estos puntos favorecerían considerar que la unidad doméstica básica estaría formada por el 
conjunto de varias familias nucleares. El resultado final expresaría, de ese modo, el crecimiento de la familia y el éxito en su reproducción, como se propone en otros contextos mediterráneos (Glowacki y Klein 2011: 410). Se evidenciaría la formación de unidades correspondientes a familias extensas, si entendemos la existencia de lazos consanguíneos, o bien, segmentos de linajes o facciones, si los lazos de unión están basados en relaciones sociales no consanguíneas.

Estas dinámicas de crecimiento y agregación son las más frecuentes no solo en los casos que hemos estudiado, sino en otros contextos reconocibles en el área de estudio. A nuestro parecer son relevantes por cuanto ayudan a entender el significado de la estructura social ibérica más allá de la unidad básica de la familia nuclear y así, comprender la configuración de la sociedad como una estructuración desde la base, con el entretejido de las unidades básicas, en un proceso de abajo a arriba que alcanza de forma escalar a unidades organizativas cada vez mayores.

\section{PROCESOS DE CONFIGURACIÓN DEL ESPACIO Y DINÁMICA SOCIAL}

La visión de los planos de asentamientos y viviendas nos ofrece la imagen final del estado del poblado tras el abandono, formación del depósito estratificado y excavación. Y la lectura que se puede ofrecer parte de la limitación de la foto fija. Sin embargo, podemos transformar esa imagen en una secuencia de la configuración del hábitat. Ello es posible en aquellos casos donde el propio proceso de ocupación ha sido lo suficientemente largo y donde el registro arqueológico ha permitido detallar esas transformaciones. Los ejemplos que hemos empleado en este estudio permiten esa lectura secuencial y ofrecer algunos comentarios de interés.

En primer lugar, podemos señalar que en los casos estudiados en ningún momento se produce un cambio del esquema espacial que rige el diseño inicial de las viviendas. Las casas con espacio de transición y convergencia añadieron nuevos departamentos a partir de su conexión a este ámbito, como en la casa IIIG de El Oral. Por su parte, la casa IIIK pudo haber transformado su esquema inicial tras la agregación de nuevos espacios, pero no lo hizo.
En segundo lugar, los espacios que se anexan al núcleo original de la casa son de naturaleza variable. En ocasiones se trata de áreas de actividad económica como almacenes, zonas de trabajo y transformación, etc. en ese sentido se podría deducir la ampliación de la capacidad económica de la unidad doméstica sobre la que se consideró necesaria en el diseño inicial. En otras ocasiones se trata de adyacencia de nuevas viviendas a la estructura inicial, lo que se puede relacionar con la ampliación del grupo doméstico, bien por el crecimiento de la familia o por la incorporación de allegados y grupos afines.

En el caso de la casa-agregado del Puig ya decíamos que puede tratarse de un rápido crecimiento del grupo doméstico que produjo la ampliación desde dos familias nucleares a fines del s. V a. C. hasta cuatro unidades en la primera mitad del s. IV a. C. (Grau y Segura 2013: 177-190). Esta duplicación de familias puede responder a un proceso de crecimiento vegetativo o natural, con el consiguiente incremento de los pobladores que son los hijos naturales de las familias originarias. En tal caso, toda la descendencia de la familia originaria pasó a habitar los nuevos espacios construidos. Pero también cabe la posibilidad de que los vínculos fuesen de carácter no consanguíneo y se relacionen con pactos y clientelas.

Nuevos ejemplos pueden añadirse a estos procesos de agregación que nos trasladan a los vínculos más allá de la estricta familia nuclear. En la ciudad ibérica de La Serreta d'Alcoi durante el s. III a. C. encontramos procesos de adyacencia que desde un punto de vista estructural señalan un paulatino crecimiento de la zona habitada. En el conocido como sector G (Llobregat, Cortell, Juan y Segura 1992) se observa claramente cómo se adosan sucesivamente hasta tres unidades de vivienda (fig. 9) que replican un idéntico esquema espacial simple y expuesto. Las nuevas viviendas se vinculan unas a otras físicamente, construyéndose aprovechando las estructuras preexistentes, de lo que se deduce algún tipo de vínculo entre ellas. En caso contrario, se hubiera conformado una manzana de casas separadas, como las que componen el bloque al sur de la calle, con muros independientes que aíslan cada vivienda.

De nuevo pudo tratarse de crecimiento vegetativo y ampliación de las familias originarias en un corto espacio de tiempo, correspondiente a una única fase del s. III a. C. Sin embargo, la rapidez de los procesos de ampliación descritos puede responder a dinámicas sociales de agregación producida por la vinculación de familias a partir de lazos de afiliación. En ese sentido 
las relaciones sociales, la cooperación en el trabajo, la utilización compartida de medios de producción y otros motivos serían la justificación de estos agregados.

Parece claro la sociedad ibérica se caracterizó por una estructura dinámica de interrelación de unidades domésticas de diverso tipo y composición. Posiblemente en estas agregaciones lo que estamos documentando es la formación de unidades suprafamiliares, que acogerían grupos biológicos amplios y también grupos afiliados sin relación consanguínea. De ese modo se abría el proceso de reconfiguración de las unidades sociales en la base y la creación de la estructura del linaje, facción o grupo. La vinculación por lazos de tipo político, como la clientela, permitiría transformar las unidades sociales anteriores y hacerlas crecer más allá de los límites estructurales que establecía la consanguinidad. Las funciones y la acumulación de riqueza aparecen reflejadas en estos conjuntos, como las manzana del asentamiento, reforzando el sentido de las agrupaciones suprafamiliares (Bonet y VivesFerrándiz 2011: 253).

Por lo tanto, podemos reconocer los procesos sociales dinámicos a partir de la transformación de los espacios domésticos, con el aumento y disminución de la importancia de unas unidades y grupos sobre otros. Así las cosas, ubicamos la agencia a nivel del grupo doméstico y podemos reconocer la importancia de las agregaciones de unidades de base en un complejo trenzado de relaciones económicas y sociales. La puesta en marcha de estas dinámicas sociales permite entender la forma en que se fueron agregando unidades cada vez mayores para constituir facciones, comunidades y territorios.

\section{VALORACIONES FINALES}

Las aproximaciones realizadas a partir de las posibilidades que ofrece el análisis de sintaxis espacial nos permiten reconocer la materialización arquitectónica de prescripciones sociales específicas, que hacían que determinada gente se comportase de una determinada forma. Los diferentes modelos de casas se generaron a partir de programas específicos escogidos por determinadas familias al construir sus viviendas. Unas quisieron dotarse de espacios liminales, mientras que otras no lo hicieron, de forma que permitieron o evitaron encuentros no deseados.

Las casas replegadas en sí mismas, con esquemas arbóreos y espacios de transición son las menos frecuentes en la región de estudio, al menos con la documentación disponible a día de hoy, y solo las encontramos en las casas del s. V a. C. del Oral. Las circunstancias socioeconómicas que condicionaron su existencia, desde el modelo de familia, la reclusión femenina ante visitantes, o las estrategias de reproducción de la familia (Grau 2013) no debieron ser extensibles a otros contextos y asentamientos. Ni siquiera en poblados con grandes similitudes como La Illeta dels Banyets de Campello.

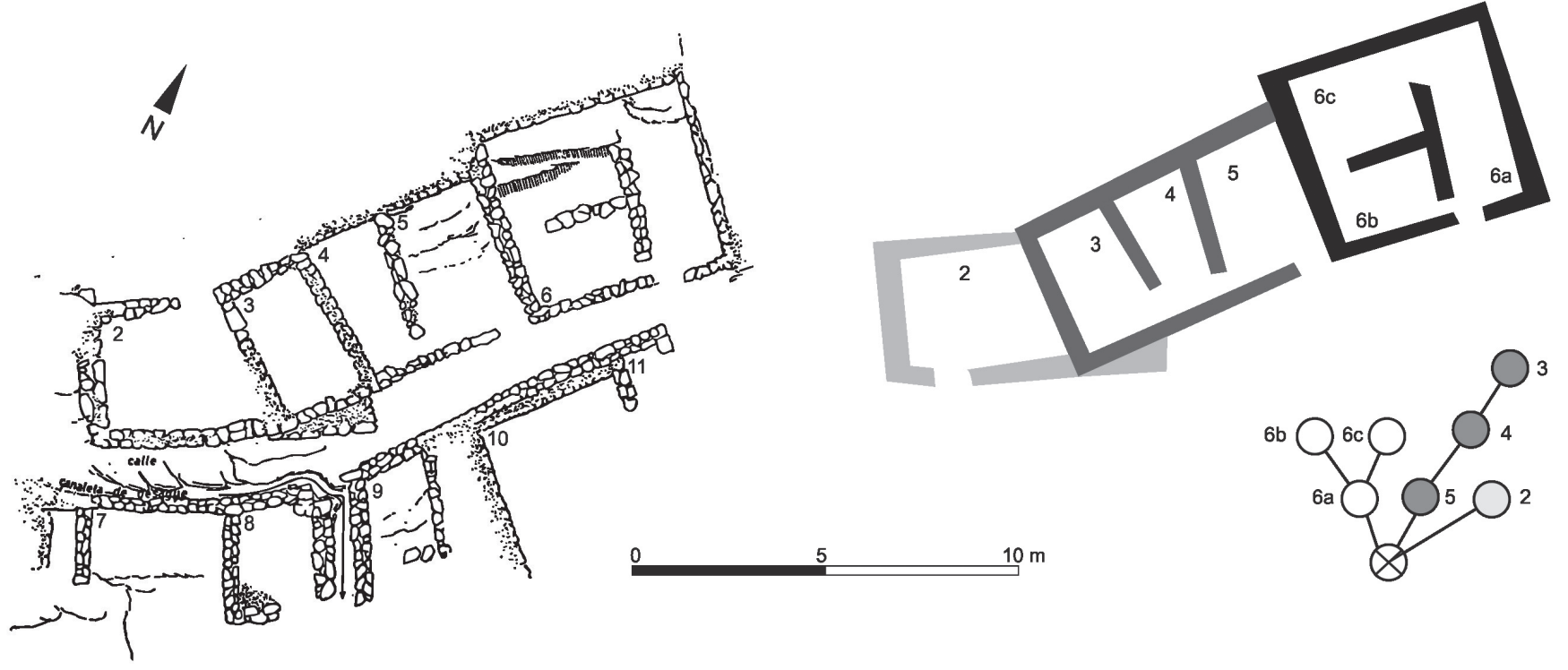

Figura 9.- Planta y mapa gamma justificado de las casas del sector G de La Serreta (Elaboración propia a partir de Llobregat, 1972). 
Por el contrario, las formas de agregación de carácter abierto y expuesto se encuentran de forma recurrente en gran cantidad de casos en el área de estudio. Los procesos que se reconocen en la configuración del hábitat posiblemente se relacionen con las dinámicas sociales de agregación de los grupos ibéricos. Este proceso produjo la forma arquitectónica que a su vez, recíprocamente, materializó, perpetuó e intensificó la complejidad social, socavando la cohesión social y acentuando la diferenciación de determinados grupos familiares sobre otros. Estos juegos de poder entre familias que acrecentaron su poderío, mientras otras se vieron abocadas a una posición subalterna, se materializaron en los modelos y formas de configuración del espacio. Nuevos y específicos estudios en este complejo campo de investigación, sin duda contribuirán a una mejor comprensión del espacio y la sociedad ibérica.

\section{FICHA TÉCNICA}

Este trabajo se ha realizado en el marco del proyecto HAR2012-37003-C03-02 del MINECO.

\section{BIBLIOGRAFÍA}

Abad, L. y Sala, F. 1993: El poblado ibérico de El Oral (San Fulgencio, Alicante), Trabajos Varios del S.I.P., 90, Dip. De Valencia, Valencia.

Abad, L., Sala, F., Grau, I., Moratalla, J., Pastor, A. y Tendero, M. 2001: Poblamiento ibérico en el Bajo Segura: El Oral (II) y La Escuera, Bibliotheca Archaeologica Hispana, 12, RAH, Madrid.

Allison, P. 1998 (Ed.): The Archaeology of Household Activities. London y New Cork.

Aranegui, C. 2012: Los Iberos, ayer y hoy. Arqueologías y culturas, Marcial Pons, Madrid.

Belarte, C. 2013: "El espacio doméstico y su lectura social en la protohistoria de Cataluña (s. VII - II/I a.C.)", en S. Gutiérrez e I. Grau, De la estructura doméstica al espacio social. Lecturas arqueológicas del uso social del espacio. Universidad de Alicante. Alicante, pp. 77-94.

Belarte, C., Bonet, H. y Sala, F. 2009: “L'espai domèstic i l'organització de la societat ibèrica: els territoris de la franja mediterrània", Arqueo Mediterrània, 11, Barcelona, pp. 93-123.

Bermejo Tirado, J. 2009: "Leyendo los espacios: una aproximación crítica a la sintaxis espacial como herramienta de análisis arqueológico", Arqueología de la Arquitectura 6, pp. 47-62.

Bermejo Tirado, J. 2013: "Análisis social de la arquitectura doméstica romana en la región del Alto Duero: una aproximación sintáctico-espacial”, en S. Gutiérrez e I. Grau, De la estructura doméstica al espacio social. Lecturas arqueológicas del uso social del espacio. Universidad de Alicante. Alicante, pp. 141-154.

Blanton, R. E. 1994: Houses and households: a comparative study, New York.

Bonet, H., Soria, L. y Vives-Ferrándiz, J. 2011: "La vida en Las casas. Producción doméstica, alimentación, enseres y ocupantes”, en H. Bonet y J. Vives-Ferrándiz, La Bastida de les Alcusses. 1928-2010, Diputación Provincial de Valencia, Valencia, pp. 139-175.
Bonet, H., Grau I. y Vives-Ferrándiz, J. 2015: "Estructura social y poder en las comunidades ibéricas de la franja central mediterránea”. Arqueomediterranea 14 , pp. 222-243.

Bourdieu, P. 1972: Esquisse d'une Théorie de la Pratique, Droz, Ginebra.

Díes, E. y Álvarez, N. 1998: “Análisis de un edificio con posible función palacial: la casa 10 de la Bastida de les Alcusses (Moixent)", en C. Aranegui (Ed.), Congreso Internacional Los Iberos Príncipes de Occidente (Barcelona, marzo, 1998), Fundación La Caixa, Barcelona, pp. 327-341.

Díes, E., Bonet, H., Álvarez, N. y Pérez Jordà, G. 1997: "La Bastida de les Alcuses (Moixent): resultados de los trabajos de excavación y restauración. Años 1990-1995", Archivo de Prehistoria Levantina, 22, pp. 215-295.

Dobres, M. A. y Robb, J. E. (eds.) 2000: Agency in Archaeology. Routledge, London y New York.

Dobres, M. A. y Robb, J. E. 2005: “'Doing' agency: introductory remarks on methodology". Journal of Archaeological Method and Theory 12(3), pp. 159-166.

Dornan, J. L. 2002: “Agency and archaeology: past, present, and future directions". Journal of Archaeological Method and Theory 9(4), pp. 303-329.

Glowacki, K. T. y Klein, N. L. 2011: "The analysis of 'Dark Age' domestic architecture: the LM IIIC settlement at Kavousi Vronda”, en A. Mazarakis Ainian (Ed.), The 'Dark Ages' Revisited: Acta of an International Symposium in Memory of William D. E. Coulson (Volos 2011), pp. 407-418.

Grau Mira, I. 2007: “Dinámica social, paisaje y teoría de la práctica. Propuestas sobre la evolución de la sociedad ibérica en el área central del oriente peninsular", Trabajos de Prehistoria, 64 2, pp. 119-142.

Grau Mira, I. 2013: "Unidad doméstica, linaje y comunidad: estructura social y su espacio en el mundo ibérico (ss. VII-I a. C.)", en S. Gutiérrez e I. Grau, De la estructura doméstica al espacio social. Lecturas arqueológicas del uso social del espacio. Universidad de Alicante. Alicante, pp. 59-76.

Grau Mira, I. y Segura Martí, J. M. 2013. El oppidum ibérico de El Puig d'Alcoi. Asentamiento y paisaje en las montañas de la Contestania, Ajuntament d'Alcoi, Alcoi.

Gutiérrez, S. y Grau, I. 2013: De la estructura doméstica al espacio social. Lecturas arqueológicas del uso social del espacio. Universidad de Alicante. Alicante.

Hanson, J. 1998: Decoding Homes and Houses, Cambridge University Press, Cambridge.

Hillier, B. 1996: Space is the Machine, Cambridge University Press, Cambridge.

Hillier, B. y Hanson, J. 1984: The Social Logic of Space, Cambridge University Press, Cambridge.

Kent, S. 1990: "A cross-cultural study of segmentation, architecture, and the use of space. New directiones in Archaeology", en S. Kent (Ed.) Domestic Architecture and the Use of Space: An Interdisciplinary Cross-Cultural Study, Cambridge, pp. 186-205.

Llobregat Conesa, E. A., Cortell Pérez, E., Juan Moltó, J. y Segura Martí, J. M. 1992: "El urbanismo ibérico en La Serreta", Recerques del Museu d'Alcoi 1: 37-70.

Olcina, M. 2005: "La Illeta dels Banyets, El Tossal de Manises y La Serreta", en L. Abad, F. Sala e I. Grau (eds.): La Contestania Iberica, treinta años después. Universidad de Alicante. Alicante, pp. 147-177.

Olcina, M., Martínez, A. y Sala, F. 2009: La Illeta dels Banyets (El Campello, Alicante). Epocas ibérica y romana, Ed. MARQ: Alicante.

Rapoport, A. 1978: Aspectos humanos de la forma urbana, Ed. Gustavo Gili, Barcelona.

Ruiz Rodríguez, A. 2007: "Los Iberos", en F. Gracia (ed.), De Iberia a Hispania, Barcelona, 733-839.

Sala, F. 2005: "Consideraciones en torno a la arquitectura y el urbanismo de la Contestania Ibérica”, en L. Abad, F. Sala e I. Grau (eds.), La Contestania Ibérica, treinta años después, Alicante, pp. 119-146.

Sala, F. y Abad, L. 2006: "Arquitectura monumental y arquitectura doméstica en la Contestania", Lucentum, 25, pp. 23-46.

Sanmartí, J. 2004. "From local groups to early states: the development of complexity in protohistoric Catalonia", Pyrenae 35, pp. 7-42. 
Sanmartí, J. y Belarte, C. 2001: Urbanización y desarrollo de estructuras estatales en la costa de Cataluña (siglos VII-III a. C.)", L. Berrocal-Rangel y Ph. Gardes (eds.), Entre celtas e iberos. Las poblaciones protohistóricas de las Galias e Hispania, Bibliotheca Archaeologica Hispana 8, Real Academia de la Historia, Madrid, pp. 161-174.
Vives-Ferrándiz, J. 2013 "Del espacio doméstico a la estructura social en un oppidum ibérico. Reflexiones a partir de la Bastida de les Alcusses", en S. Gutierrez e I. Grau (eds.), De la estructura doméstica al espacio social. Lecturas arqueológicas del uso social del espacio, Universidad de Alicante, Alicante, pp. 95-111. 